\title{
Dietary sodium intake and its relationship to adiposity in young black and white adults: The African-PREDICT study
}

\author{
Simone H. Crouch MHSc ${ }^{1}$ (D) | Lisa J. Ware PhD ${ }^{1}$ (D) | Lebo F. Gafane-Matemane PhD ${ }^{1,2}$ \\ (D) | Herculina S. Kruger PhD ${ }^{2,3}$ (D) Tertia Van Zyl PhD ${ }^{3}$ (D) | Bianca Van der Westhuizen \\ $\mathrm{PhD}^{1}$ (D) | Aletta E. Schutte PhD ${ }^{1,2}$
}

${ }^{1}$ Hypertension in Africa Research Team (HART), North-West University, Potchefstroom, South Africa

${ }^{2}$ MRC Research Unit: Hypertension and Cardiovascular Disease, North-West University, Potchefstroom, South Africa

${ }^{3}$ Centre of Excellence for Nutrition, NorthWest University, Potchefstroom, South Africa

\section{Correspondence}

Aletta E. Schutte, Hypertension in Africa Research Team (HART), North-West University, Potchefstroom, South Africa. Email: alta.schutte@nwu.ac.za

\section{Funding information}

The research funded in this manuscript is part of an ongoing research project financially supported by the South African Medical Research Council (SAMRC) with funds from National Treasury under its Economic Competitiveness and Support Package; the South African Research Chairs Initiative (SARChI) of the Department of Science and Technology and National Research Foundation (NRF) of South Africa [UID:86895]; the Strategic Health Innovation Partnerships (SHIP) Unit of the SAMRC with funds received from the South African National Department of Health, GlaxoSmithKline R\&D, the UK Medical Research Council and with funds from the UK Government's Newton Fund; as well as corporate social investment grants from Pfizer (South Africa), BoehringerIngelheim (South Africa), Novartis (South Africa), the Medi Clinic Hospital Group (South Africa), and in kind contributions of Roche Diagnostics (South Africa). Any opinion, findings and conclusions, or recommendations expressed in this material are those of the authors and therefore, the NRF does not accept any liability in this regard.
Obesity and salt intake are both established factors contributing to cardiovascular disease development. Recently, studies found a controversial positive relationship between dietary salt and obesity. Therefore, the authors investigated whether obesity-related measures are associated with 24-hour urinary sodium in a healthy biethnic population. The study included 761 adults (20-30 years) with complete 24 -hour urinary sodium, anthropometry, and bioelectrical impedance measurements. In single regression analyses all obesity-related measures related positively with 24-hour urinary sodium $(P \leq .008)$. However, with multivariate adjustments for energy intake, accelerometery, age, sex, black and white ethnicity, and other covariates, only body surface area (BSA) remained independently associated with 24-hour urinary sodium $\left(R^{2}=0.72, \beta=.05, P=.039\right)$. To conclude, we found a consistent and robust positive relationship between BSA and estimated salt intake - but not with traditional obesity measures such as body mass index (BMI). Further studies are needed to investigate body surface area and potentially, skin area, in salt handling. 


\section{1 | INTRODUCTION}

Obesity is a growing public health concern. Several factors are known to contribute to obesity, but recently, studies found a positive link between dietary salt and obesity. ${ }^{1-6}$ However, the nature of this relationship remains a matter of debate. As both obesity as sodium intake are targeted by public health initiatives, the potential for a relationship between the two is noteworthy. Compared to individuals on lower sodium diets, those with a high sodium diet have a $20 \%$ higher risk of central obesity. ${ }^{1,5}$ The American Heart Association suggests a maximum sodium intake of $2300 \mathrm{mg} / \mathrm{d}^{7}{ }^{7}$ which corresponds to 6 grams of salt per day - slightly higher than the World Health Organization (WHO) recommendation of $5 \mathrm{~g} / \mathrm{d} .{ }^{8}$ Some studies suggest the relationship between salt and obesity is simply the result of additional energy consumption that accompanies a high salt diet, ie, increased consumption of high energy-dense processed foods and sugar-sweetened beverages. ${ }^{2,6}$ However, others suggest salt may contribute to obesity independent of energy intake via increased extracellular fluid (increased weight via water retention), ${ }^{9}$ genetic predisposition, ${ }^{10}$ salt sensitivity, ${ }^{11}$ or altered fat metabolism. ${ }^{5}$

Both high sodium intake and obesity are independent risk factors for hypertension and cardiovascular disease. ${ }^{12,13}$ A relationship between sodium intake and obesity suggests an additional cardiovascular risk associated with high dietary sodium. However, the existing literature demonstrating a relationship between dietary sodium intake and obesity contains important limitations, including the failure to take several key potential confounders into consideration, such as energy expenditure, glucose, and aldosterone.

In addition, few studies included a young healthy adult population without conditions that may influence the sodium/obesity relationship. ${ }^{1-4}$ Lastly, most studies evaluate only 1 or 2 obesity-related markers whereas we evaluated several measures. ${ }^{1-6}$ We therefore investigated the relationship between a range of measures reflecting obesity and overall body composition and sodium intake (24-hour urinary sodium excretion) while controlling for energy intake, expenditure, and other potential confounders in apparently healthy, young black and white adults.

\section{2 | METHODS}

\section{1 | Study population}

This study forms part of the African-PREDICT study (African Prospective study on the Early Detection and Identification of Cardiovascular disease and Hypertension), which recruited and monitored young adults over $10-20$ years. The inclusion criteria for this study were black or white, apparently healthy individuals, aged 20-30 years; HIV uninfected; office blood pressure $<140 \mathrm{~mm} \mathrm{Hg}$ systolic and < $90 \mathrm{~mm} \mathrm{Hg}$ diastolic; no previous diagnosis or medication for a chronic disease; not pregnant or lactating. This study used cross-sectional data from the first 761 participants with complete data sets. This study was approved by the Health Research Ethics Committee (HREC) of the North-West University (NWU-00050-17A1) and adheres to the Declaration of Helsinki, and all participants in the study provided written informed consent prior to taking part in the study.

\section{2 | General health and demographic questionnaire}

Data with regard to age, sex, and ethnicity were collected using a General Health and Demographic Questionnaire.

Socioeconomic status was calculated using a point system adapted to the South African context from the Kuppuswamy's Socioeconomic Status Scale 2010. ${ }^{14}$ The adapted version scored participants in three categories: (1) skill level, (2) education, and (3) household income.

\section{3 | Twenty four hours urine collection}

Participants were instructed to collect a 24-hour urine sample on a day that was convenient for them. The first urine of the day was discarded and all urine passed thereafter was collected, including the first urine of the following morning (day 2). Start and finish times were recorded. The protocol for 24-hour urine collection follows that of the Pan American Health Organization/World Health Organization (PAHO/WHO) protocol. ${ }^{15}$ Incomplete urine collections were defined as a volume less than $300 \mathrm{~mL}$ per 24 hours and/or a 24-hour creatinine excretion of $<4 \mathrm{mmol}$ or $>25 \mathrm{mmol}$ in women and $<6 \mathrm{mmol}$ or $>30 \mathrm{mmol}$ in men.

\section{4 | Body composition measurements}

Trained researchers measured weight $(\mathrm{kg})$ (SECA electronic scales, SECA, Birmingham, UK) and height $(m)$ (SECA stadiometer, SECA, Birmingham, UK). Waist, hip, and neck circumferences were measured 3 times using an anthropometric nonflexible tape measure (Holtain, Ltd, Crymych, UK) and the median values used. Body mass index (BMI) was calculated using the standard weight (kg)/ height $\left(\mathrm{m}^{2}\right)$ formula, and waist-to-height ratio and waist-to-hip ratio were calculated (overweight BMI $25-29.9 \mathrm{~kg} / \mathrm{m}^{2}$; obese, BMI $>30 \mathrm{~kg} / \mathrm{m}^{2}$ ). ${ }^{16}$ Body surface area (BSA) was determined using the Mosteller formula. ${ }^{17}$ All measurements were performed following the guidelines of the International Society for the Advancement of Kinanthropometry.

Bioelectrical impedance measurements were performed to estimate lean body mass, water, and body fat percentage using a Bodystat 1500MDD dual-frequency analyser (Bodystat, Ltd, Ballakaap, UK) after 8 hours fasting and with an empty bladder. Participants were in a supine position while electrodes were placed on the skin of the individual's right hand and foot. A low-level, battery-generated electrical current was passed through the body and the impedance (at frequencies of $5 \mathrm{kHz}$ and $50 \mathrm{kHz}$ ) measured. Studies have validated bioelectrical impedance in the estimation of percentage body fat compared with dual-energy X-ray absorptiometry. ${ }^{18}$ 


\section{5 | Twenty-four hour dietary recall questionnaire}

A 24-hour dietary recall questionnaire was administered by a dietician on the study day and on 2 subsequent days to provide an analysis of each participant's dietary intake on 2 weekdays and 1 weekend day. Energy and nutrient intake was calculated using the South African Food Composition database ${ }^{19}$ and the individual average intakes were calculated from the 3 recorded days.

\subsection{Ambulatory blood pressure}

Participants were fitted with a validated 24-hour ambulatory blood pressure monitor (ABPM) (CardioXplore ${ }^{\circledR}$ CE120, Meditech, Budapest, Hungary). The apparatus was programmed to take recordings every 30 minutes during the day (06:00 to 22:00 hours) and every hour during the night (22:00 to 06:00 hours). The ABPM was fitted to each participant at approximately the same time every day (late morning), using an appropriate sized cuff fitted to the participant's nondominant arm. Only participants with $>70 \%$ valid 24 -hour blood pressure measurements and $>20$ daytime measurements and $>7$ nighttime measurements were included in the data analysis following the criteria recommended by the European Society of Hypertension.

\section{7 | Physical activity measurements}

The ActiHeart device (CamNtech Ltd., England, UK), designed to capture heart rate variability and movement using a triaxial accelerometer, was worn for a maximum of 7 days and average daily total and activity energy expenditure (total energy expenditure [TEE], activity energy expenditure [AEE]) were calculated. ${ }^{20}$ The Global Physical Activity Questionnaire (GPAQ), developed by the WHO, ${ }^{21}$ was administered to measure self-reported leisure and work-related physical activity and sedentary time.

\section{8 | Blood sampling and biochemical analyses}

Participants were asked to fast overnight for at least 8 hours. Venous blood samples were collected by a qualified nurse. The samples were prepared according to standardized protocols and stored at $-80^{\circ} \mathrm{C}$.

Serum samples were analyzed for C-reactive protein, creatinine, total cholesterol, high- and low-density lipoprotein cholesterol (HDL-C, LDL-C), glucose, and gamma glutamyltransferase (GGT) (Cobas Integra 400 plus, Roche, Basel, Switzerland). Serum leptin, adiponectin, and renin were determined using Quantikine ELISA kits (R\&D Systems, Minneapolis, MN) and aldosterone was detected using the RIA Aldosterone Kit (Beckman Coulter, Immunotech, Radiova, Czech Republic). Serum cotinine was analyzed using a chemiluminescence method on the Immulite (Siemens, Erlangen, Germany) apparatus. Estimated glomerular filtration rate (eGFR) was calculated using the Chronic Kidney Disease Epidemiology (CKD-EPI) formula. ${ }^{22}$ Urinary sodium, potassium, and chloride were measured by means of ion-selective electrode potentiometry on the Cobas
Integra ${ }^{\circledR} 400$ plus and creatinine concentrations were measured using the Creatinine Jaffé Gen.2 reagent (Roche, Basel, Switzerland). Daily urinary sodium and potassium excretion $(\mathrm{mmol} / \mathrm{d})$ were calculated by multiplying the sodium, potassium, and creatinine concentrations ( $\mathrm{mmol} / \mathrm{L}$ ) of the 24-hour urine by the total 24-hour volume of urine (in liters). Daily salt intake was estimated from 24-hour urinary sodium excretion by converting sodium in mmol to $\mathrm{mg}$ : sodium $(\mathrm{mmol}) \times 23=$ sodium $(\mathrm{mg})^{23}$ and then applying the conversion: $1 \mathrm{~g}$ salt (sodium chloride) $=390 \mathrm{mg}$ sodium ${ }^{23}$

\section{9 | Statistical analysis}

Statistica v13.2 (Dell Software, Round Rock, TX; 2016) was used for data analysis. All continuous variables were checked for normality by visual inspection (quantile-quantile plots) and the KolmogorovSmirnov test. Variables with non-Gaussian distributions were logarithmically transformed. Interactions of sex, ethnicity or socioeconomic status on the relationship between BMI, BSA, waist circumference, hip circumference, neck circumference, waist-to-hip ratio, waist-to-height ratio, body fat \%, lean mass, leptin and adiponectin and 24-hour urinary sodium excretion were investigated. To ensure sufficient sample size, a power analysis was performed using the G*Power 3 statistical analysis program. This indicated a minimum sample size of $n=89$ is required (for an effect size of $f^{2}=0.15, \alpha$ err prob $=0.05$, max covariates $=12$ ). $T$-tests and chisquare tests were performed to compare the profiles of black and white men and women. The relationships between obesity-related measures as dependent variables and 24-hour urinary sodium excretion (as the main independent variable) were explored using Pearson, partial, and multiple regression analyses. In Model 1 we adjusted for TEE only, in Model 2 energy intake only, and in Model 3 both TEE and energy intake. In Model 4 we aimed to replicate the model by $\mathrm{Ma}$ and colleagues ${ }^{1}$ and adjusted for age, socioeconomic score, total energy expenditure, dietary energy intake, and self-reported tobacco and alcohol use. Model 5 was adjusted for age, socioeconomic score, TEE, energy intake, cotinine, GGT, HDL-C, systolic ambulatory blood pressure, glucose, C-reactive protein, and aldosterone. The covariates for Model 5 were determined based on bivariate correlations with the dependent variables and 24-hour urinary sodium excretion.

\section{3 | RESULTS}

As we found an interaction with sex between 24-hour urinary sodium and BSA $(P=.019)$ and an interaction with ethnicity between 24-hour urinary sodium and neck circumference $(P=.021)$, data are presented for the total group and additionally, by sex and ethnicity. No further interaction effects were identified (Table S1).

In Table 1, the total group consumed on average $7.65 \mathrm{~g} / \mathrm{d}$ of salt with $79.9 \%$ consuming above the daily recommended salt intake of $5 \mathrm{~g} / \mathrm{d}$, according to 24 hours sodium excretion. White men had the highest mean salt intakes (mean $8.35 \mathrm{~g} / \mathrm{d}$ ), with $84 \%$ consuming above the daily recommended intake. Although there was no 


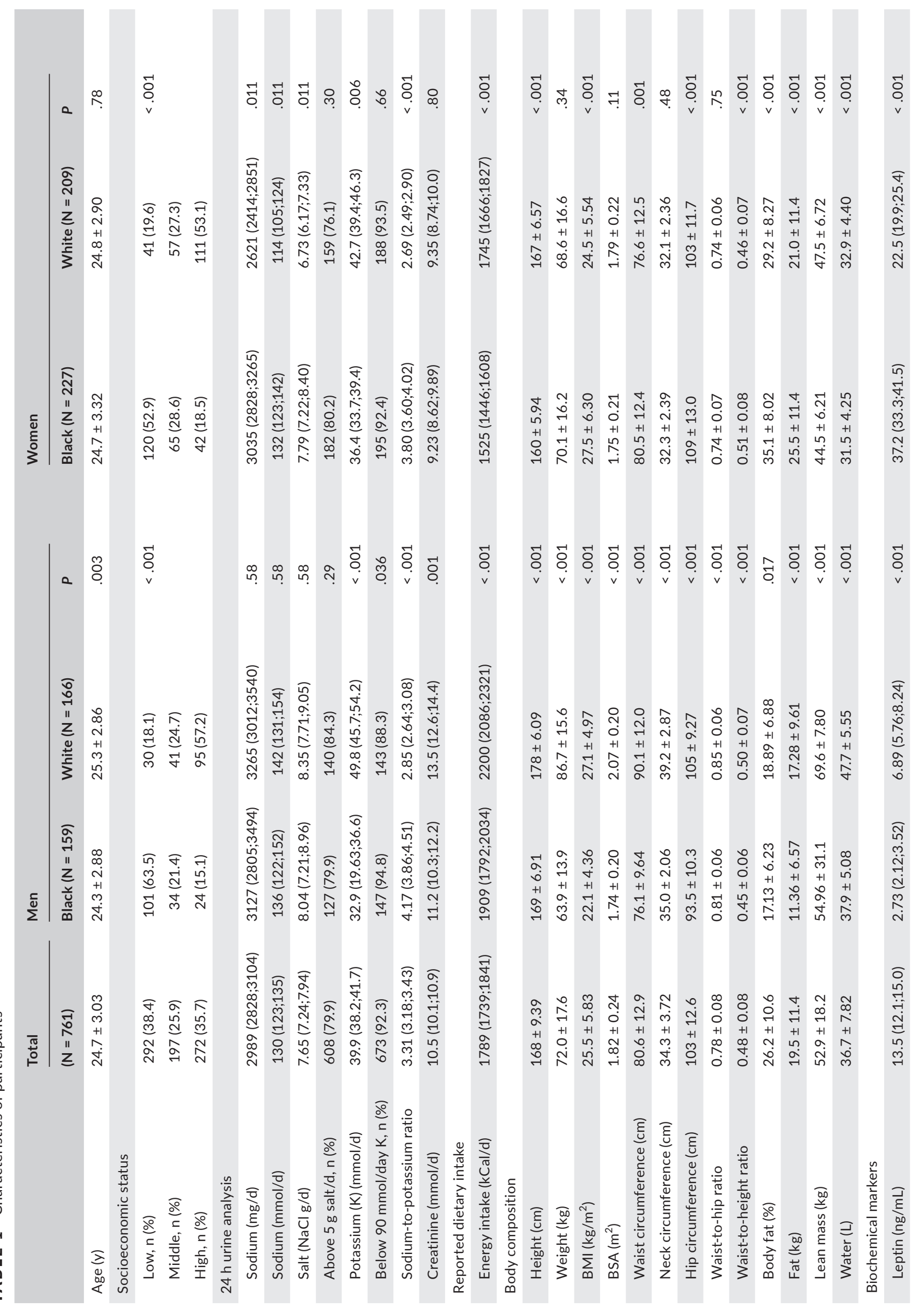




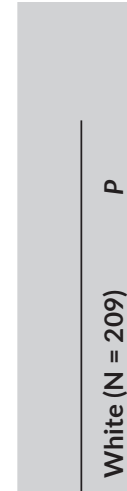

aุ

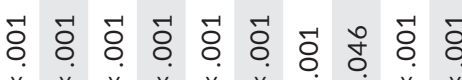

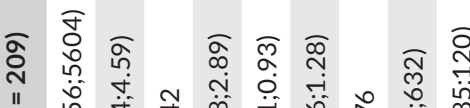

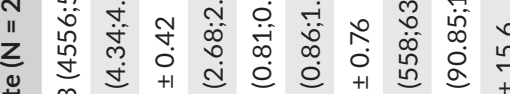

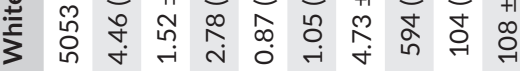

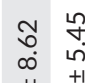

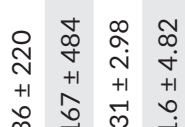

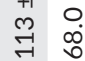

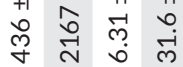

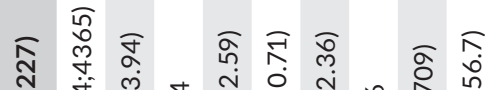

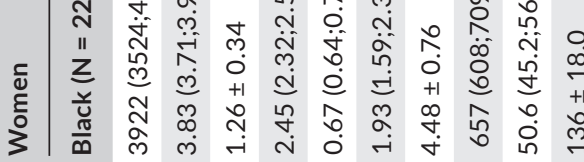

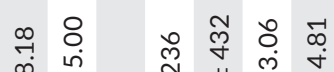

$\begin{array}{ccc}2 & 8 \\ 0 & 1 & +1 \\ +1 & +1\end{array}$

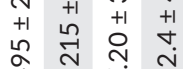

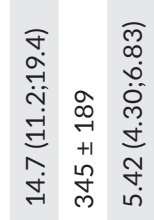

ஓ.

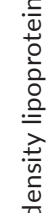

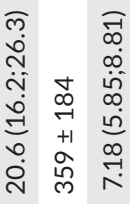

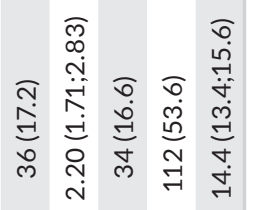

그

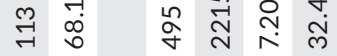

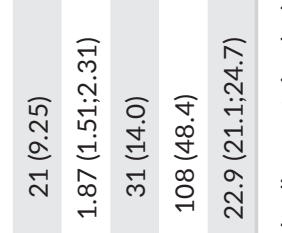

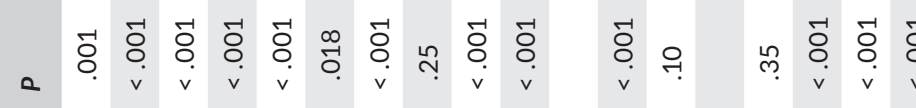

จิ ন্

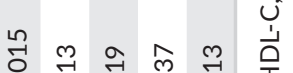

金亭

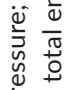

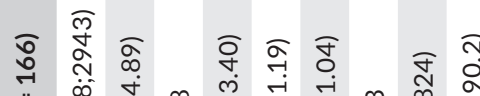

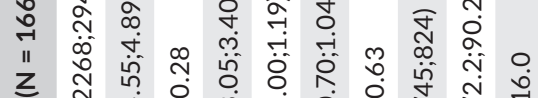

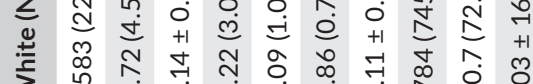

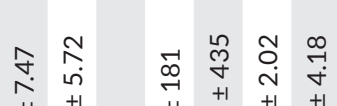

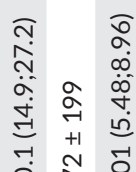

뭉

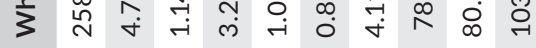

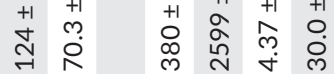

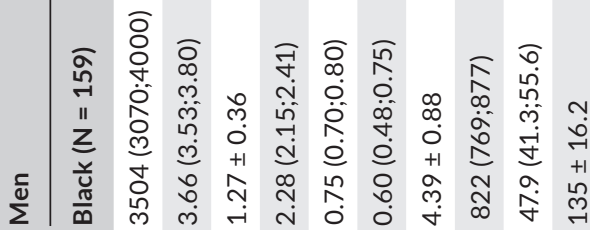

m

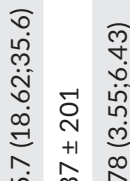

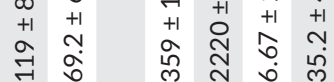

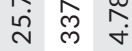

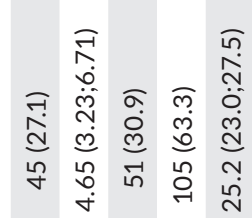

음

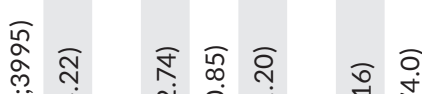

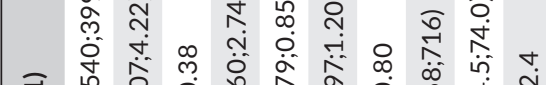

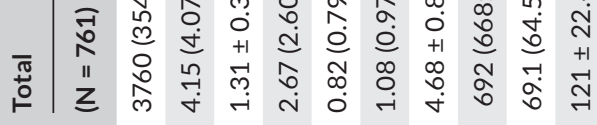

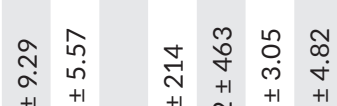

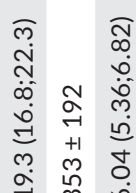

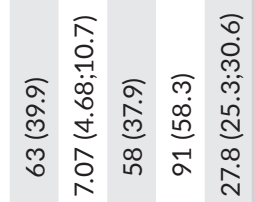

总

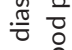

离 응

选 部

के

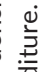


difference in 24-hour sodium excretion between black and white men, black women had higher sodium excretion than white women $(P=.011)$. Both white men and women had higher 24-hour urinary potassium than their black counterparts (both $P<.01$ ). A higher percentage of black men (94.8\%) had a 24-hour urinary potassium level below the recommended $90 \mathrm{mmol} / \mathrm{d},{ }^{24}$ than white men (88.3\%; $P=.036)$ and both black men and women had a higher sodium-topotassium ratio than whites $(P<.001)$. In contrast to these findings, analysis of dietary intake data showed that white men and women reported higher mean daily consumption energy when compared to black men and women $(P<.001)$.

In the total population, $46 \%$ were classified as overweight or obese ( $26 \%$ overweight, $20 \%$ obese). In terms of body composition, white men had higher mean values than black men on all measured variables, including height, weight, BMI, BSA, waist circumference, hip circumference, neck circumference, waist-to-hip ratio, waist-toheight ratio, fat mass, lean mass, water, and body fat percentage (all $P \leq$.017). Leptin concentrations were also higher and adiponectin lower than black men (both $P \leq .001$ ). White women showed greater height, lean mass, water, and adiponectin, with lower BMI, hip circumference $(P \leq .001)$, and renin $(P=.046)$ than black women. When objectively measured energy expenditure was calculated per kilogram body weight to adjust for differences in body size, black men presented with higher AEE/kg and TEE/kg $(P<.001)$ than white men. No differences were observed between black and white women.

Comparing self-reported health behaviors, black men reported more tobacco use than white men $(P=.015)$ whereas white women reported more tobacco use than black women $(P=.014)$, though no significant differences were observed in serum cotinine levels. Self-reported alcohol intake also did not differ between the groups, though the nonspecific marker GGT was higher in white than in black women $(P<.001)$.

To address our aim, we performed single regression analysis between obesity measurements and 24-hour urinary sodium as well as sodium-to-potassium ratio (Tables S2 and S3). No consistently significant findings were found between obesity measures and sodiumto-potassium ratio. The 24 -hour urinary sodium correlated positively with all anthropometric measures in the total group $(P \leq .008)$; and with several measures in the black and white men, namely weight, BMI, BSA, waist circumference, waist-to-hip ratio, and waist-toheight ratio (all $P<.05$ ), but with no anthropometric measures in black or white women. A negative correlation was present between 24-hour urinary sodium and adiponectin only in black women $(P=.001)$. In the single regression analyses, the most prominent and consistent results were observed between BSA and 24-hour sodium.

Multivariate-adjusted regression analysis (Table 2) of associations between either BSA or BMI and 24-hour urinary sodium showed both BSA and BMI associated positively with 24-hour urinary sodium in the total group, black men, and white men (unadjusted model; all $P \leq$.032) but not in women. In Model 1, adjusted for TEE only, BSA associated positively with 24-hour urinary sodium in both the total group and white women (all $P \leq .037$ ). Following adjustment for energy intake only (Model 2), BSA remained associated with 24-hour urinary sodium in the total group and additionally in black and white men (all $P \leq .012$ ), BMI also associated positively with 24-hour urinary sodium in the total group and white men (both $P \leq$.026). Model 3 , adjusted for both TEE and energy intake, showed an association between BSA and 24-hour urinary sodium in the total group only $(P=.005)$. In Model 4, we wanted to replicate the analysis of $\mathrm{Ma}$ and colleagues, ${ }^{1}$ who found a positive relationship between $\mathrm{BMI}$ and sodium intake when adjusting for age, socioeconomic score, TEE, dietary energy intake, and self-reported tobacco and alcohol use. We also found a significant positive association but only between BSA and 24-hour urinary sodium in the total group and white women (both $P \leq .043$ ). This model was chosen as it most closely resembled our aim. Lastly, we ran a fully adjusted model including systolic blood pressure and a range of additional covariates (age, socioeconomic status, cotinine, GGT, aldosterone, C-reactive protein, LDL-C, HDL-C and glucose; Model 5). In this model, BSA, but not BMI, remained significantly associated with 24-hour urinary sodium in the total group $(P=.039)$. In all models adjusted for TEE, BMI was no longer significantly associated with 24 -hour urinary sodium.

Within the total group when using the fully adjusted model, we additionally tested for associations between height, weight, waist circumference, hip circumference, neck circumference, waist-to-hip ratio, waist-to-height ratio, fat mass, lean mass, body fat percentage, adiponectin, and leptin with 24-hour urinary sodium using the same models and no consistent independent associations were found (Figure).

\section{DISCUSSION}

Our aim was to evaluate if objectively measured salt intake is associated with obesity in a large, young, healthy adult cohort. We included a comprehensive range of body composition measures and determined the associations thereof with 24-hour urinary sodium. A significant positive relationship between 24-hour urinary sodium and BMI (as well as other traditional obesity measurements) was apparent in unadjusted models but was no longer observed after controlling for energy expenditure. However, the relationship between BSA and sodium was robust. This link was independent of energy intake, energy expenditure, age, socioeconomic status, and self-reported tobacco and alcohol use plus several, potentially important biochemical confounders.

Previous findings on the relationship between sodium and obesity, including a study by Ma and colleagues ${ }^{1}$ (replicated in our Model 4) showed a positive association between BMI and sodium intake, whereas BSA was not considered. ${ }^{1,3,4}$ In our analysis replicating this study, ${ }^{1}$ the link between sodium and BMI reached only borderline significance but disappeared in all models where we adjusted for objectively measured energy expenditure. BSA and sodium intake, however, remained associated even after adjustment for all covariates in the final model.

Despite the known limitations in the accurate estimation of BSA using different formulae in different individuals (obese, tall, short), ${ }^{25}$ 


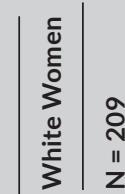

oิ

ò

ก

คิ

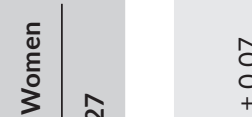

1
0
0
0
+1
0

0
0
0
0
0
0
0

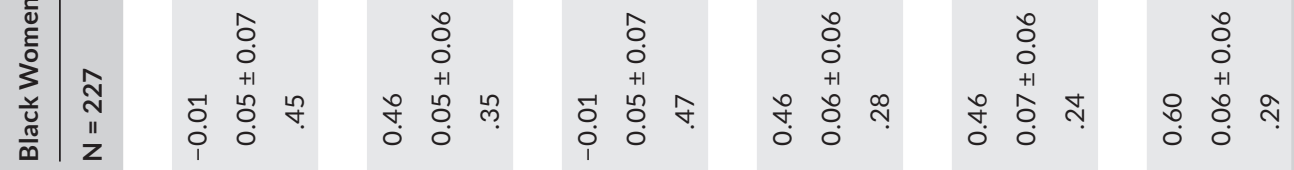

옹

1
0
0
0
+1
+1

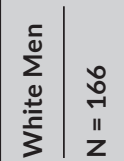

$\stackrel{\infty}{\circ}$

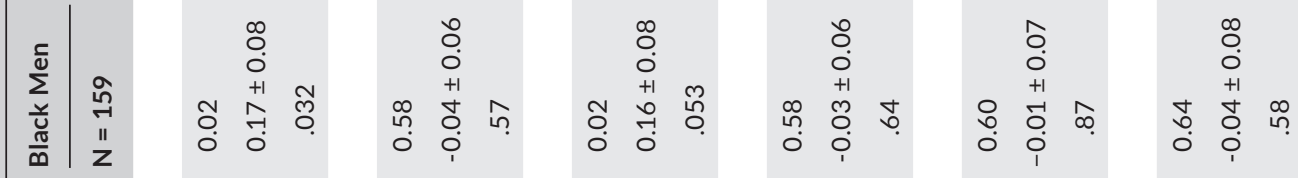

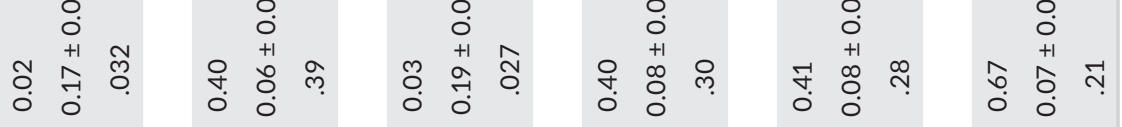
$+1$

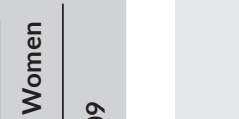

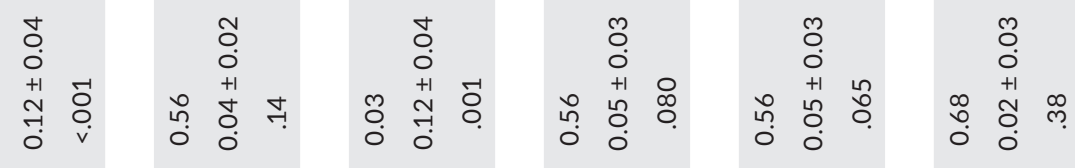

ڤ

辛

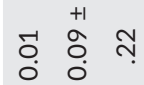

นn.

oิ

1
0
0
+1
0

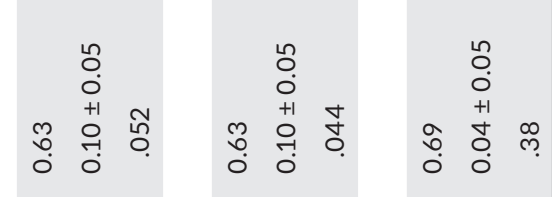

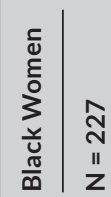

oิ̀

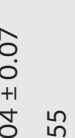

$\begin{array}{lll}0 & 0 \\ 0 & 0 & 0 \\ 0 & 0 \\ 0 & 0 \\ +1 & +1\end{array}$

$\begin{array}{ll}0 & \\ 0 & \\ 0 & \\ +1 & 0 \\ 0 & 0 \\ 0 & n\end{array}$

है

茯。

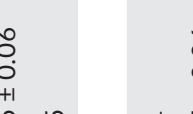

0
0
0
0
+1

‥

훙

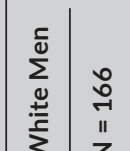

$\infty$
0
0
0
+1
+1

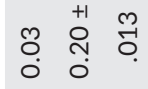

$\hat{0}$
0
0
+1
0

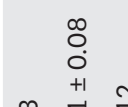

० 0

文

을

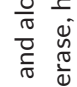

乡 $z$

ले

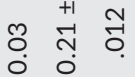

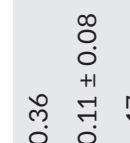

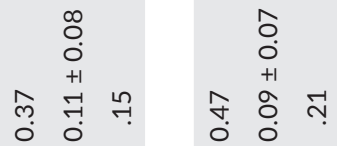

茅

항

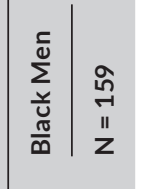

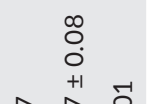

2
0
0
0
+1
+1

$\stackrel{\circ}{\circ}$

○ำ

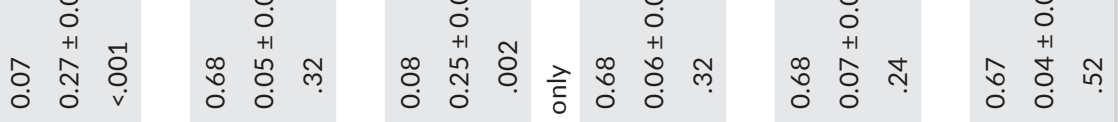

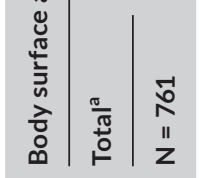

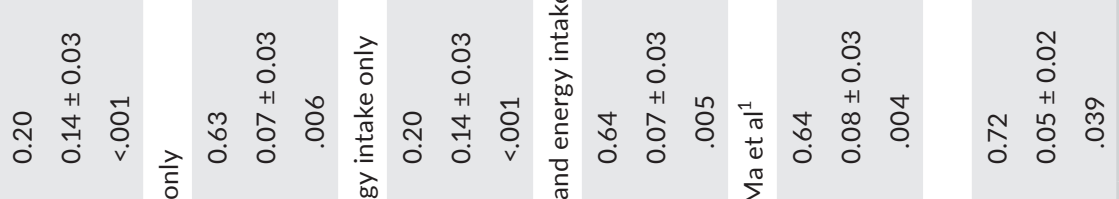

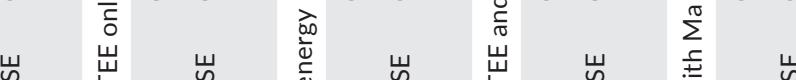

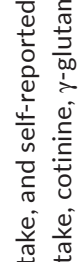

$\stackrel{\text { वे }}{\subseteq}$

离这

业迹

券

竞

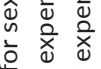

के के

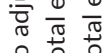

\& $\begin{aligned} & +1 \\ & +1\end{aligned}$

党

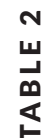

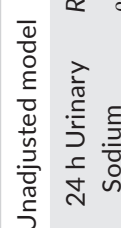
$\frac{\sqrt{2}}{\frac{1}{2}}$

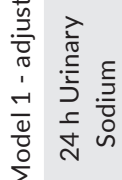

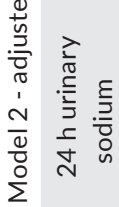

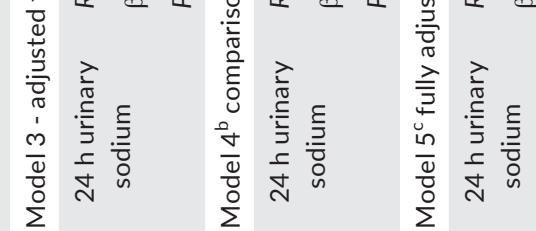

$\therefore$ 은 


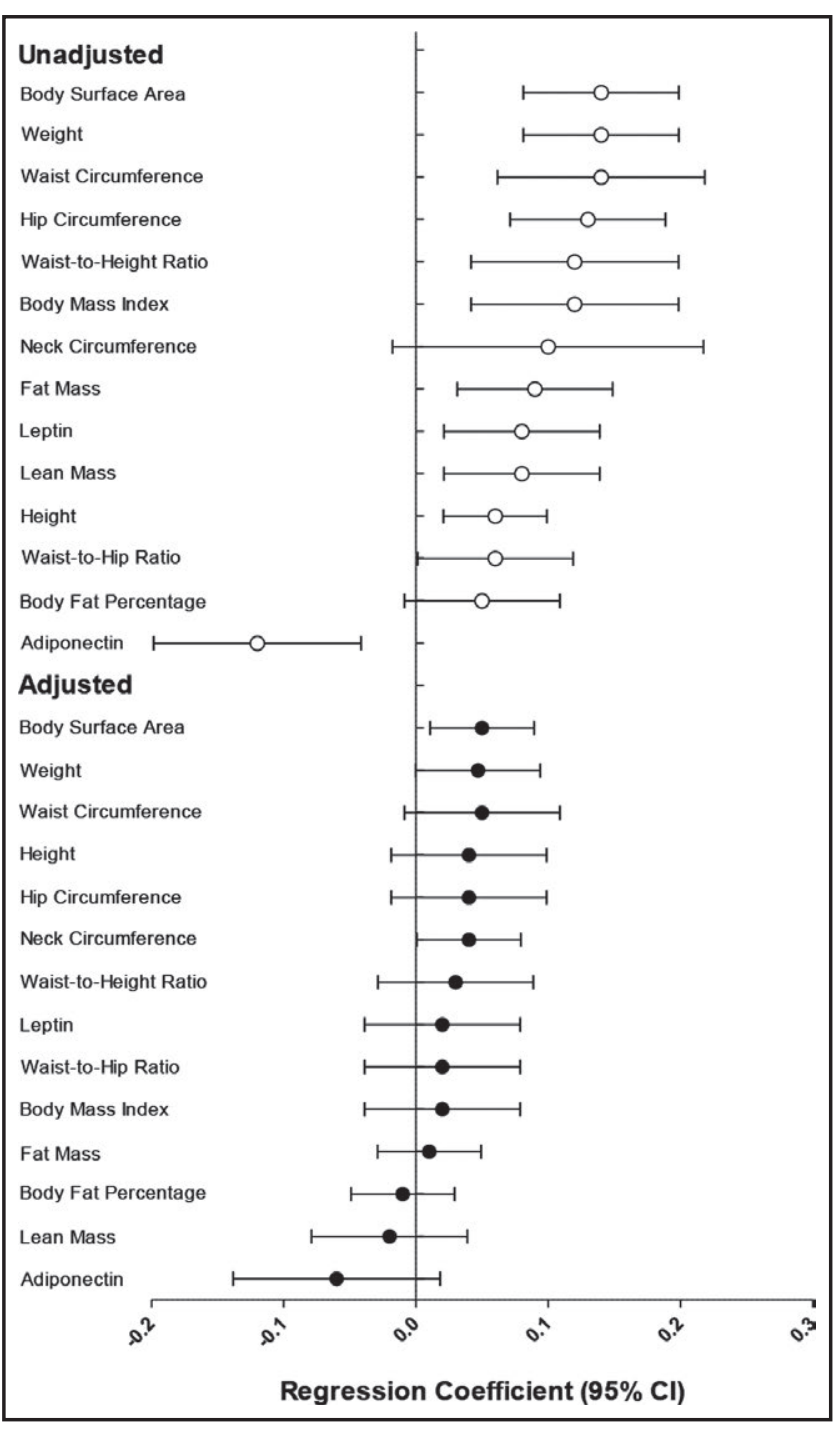

FIGURE 1 Regression coefficients for the relationship between obesity-related measures and $24 \mathrm{~h}$ urinary sodium in unadjusted and adjusted models

the question could rather be posed: is BSA a better reflection of obesity or of total skin area? This may be particularly salient as none of the more traditional measures of obesity (height, weight, BMI, waist circumference, hip circumference, neck circumference, waist-to-hip ratio, waist-to-height ratio, fat mass, lean mass, body fat percentage, adiponectin, and leptin) showed an independent association with salt intake. Further support is found for the hypothesis that BSA may reflect skin area, as it is often used to calculate the degree of damage and make medical decisions in the treatment of patients with burn injuries. $^{26}$

If BSA is indeed a marker for skin area, our findings may highlight the recent reports from the research group of Jens Titze that found sodium to be stored in the skin. ${ }^{27,28}$ During sodium loading, skin extracellular matrix molecules are modified allowing for a higher sodium storage capacity. ${ }^{29}$ Similarly during long-term low sodium diets the sodium in the skin is released, ${ }^{30}$ suggesting that the skin acts as a sodium storage facility. It was also found that tissue sodium storage was closely linked to essential hypertension. ${ }^{28}$ As yet, it is unclear if a greater BSA indicates a larger skin surface area available for greater sodium storage and further research is required to understand this relationship.

Consideration of more of the potential confounders on the relationship between sodium and obesity is important and is a limitation of many previous studies. For example, tobacco use is known to have a negative association with obesity ${ }^{31}$ and may also be related to poor diet quality and increased salt intake. ${ }^{32,33}$ The inclusion of serum cotinine as a marker of tobacco exposure in our final model is of particular importance with one quarter of our study population classified as tobacco exposed (serum cotinine $>10 \mathrm{ng} / \mathrm{mL}$ ). ${ }^{34}$ Additionally aldosterone may be related to obesity independent of salt intake. ${ }^{35}$ Yet, our finding on a positive link between 24-hour urinary sodium and BSA was independent of these and many other potential confounders.

Our study results should be interpreted within the framework of the strengths and limitations. This study was performed using a biethnic group of young, healthy men and women. Participants were screened prior to participation using strict exclusion criteria for conditions that may affect the results. In terms of strengths, the absence of preexisting chronic disease afforded us the opportunity to investigate the physiology behind this interaction in adults without influence from pathology. Additionally, our study includes multiple potential confounders such as aldosterone - not considered in other studies. ${ }^{1-4}$ Furthermore, a large number of obesity reflecting markers were considered. The large biethnic cohort used in this study allowed us to further investigate the relationship between objectively measured sodium and obesity in both a white and black population, which are reported to differ in frequency of salt sensitivity, ${ }^{36}$ as well as in body composition and diet. ${ }^{37,38}$ However, within our study we did not expect to find differences in the association between salt and obesity by sex or ethnicity as the initial interaction effects found were minimal. In terms of limitations, the potential for underreporting when using self-reported dietary intake data is well known. Although methods are available to correct for this misreporting, others have suggested this correction is unnecessary when studies include objective energy expenditure measurement. ${ }^{39} \mathrm{~A}$ further limitation is the cross-sectional study design, precluding the assessment of cause and effect. This population will have longitudinal data to address this research question in future. Additionally, the study involves only young adults. However, with almost half of the study cohort being overweight or obese and 4 out of 5 eating too much salt, it could be argued that targeting salt and obesity reduction strategies toward young adults, where interventions may still reduce disease risk, could yield the greatest potential benefit. Additionally, the use of single collection 24-hour urine for sodium analysis does not account for day-to-day variations in sodium excretion.

In conclusion, in young healthy adults we found a consistent and robust positive relationship between BSA and 24-hour urinary sodium, independent of energy intake, energy expenditure, and numerous other potential confounders. The relevance of this finding in terms of obesity remains unclear as salt intake was not independently associated with any of the traditional obesity markers 
(height, weight, waist circumference, hip circumference, neck circumference, waist-to-hip ratio, waist-to-height ratio, fat mass, lean mass, body fat percentage, adiponectin, and leptin) in the total group. Further studies are needed to investigate the association between BSA, salt intake, and skin sodium storage.

\section{ACKNOWLEDGMENTS}

The authors are grateful towards the research participants, support and research staff as well as students at the Hypertension Research and Training Clinic at the North-West University.

\section{CONFLICT OF INTEREST}

All authors declare no conflict of interest.

\section{ORCID}

Simone H. Crouch iD http://orcid.org/0000-0003-1911-5134

Lisa J. Ware iD http://orcid.org/0000-0002-9762-4017

Lebo F. Gafane-Matemane (iD http://orcid.org/0000-0003-4596-7218

Herculina S. Kruger iD http://orcid.org/0000-0002-5365-1777

Tertia Van Zyl iD http://orcid.org/0000-0001-7666-2500

Bianca Van der Westhuizen (ID http://orcid.org/0000-0003-2150-4331

Aletta E. Schutte iD http://orcid.org/0000-0001-9217-4937

\section{REFERENCES}

1. Ma Y, He FJ, MacGregor GA. High salt intake. Hypertension. 2015;115:05948.

2. Larsen SC, Ängquist L, Sørensen TI, et al. 24 hours urinary sodium excretion and subsequent change in weight, waist circumference and body composition. PLoS One. 2013;8:e69689.

3. Libuda L, Kersting M, Alexy U. Consumption of dietary salt measured by urinary sodium excretion and its association with body weight status in healthy children and adolescents. Public Health Nutr. 2012;15:433-441.

4. Zhu H, Pollock NK, Kotak I, et al. Dietary sodium, adiposity, and inflammation in healthy adolescents. Pediatrics. 2014;133:635-642.

5. Moosavian SP, Haghighatdoost F, Surkan PJ, et al. Salt and obesity: a systematic review and meta-analysis of observational studies. Int J Food Sci Nutr. 2016;68(3):1-16.

6. Grimes CA, Riddell LJ, Campbell KJ, et al. Dietary salt intake, sugarsweetened beverage consumption, and obesity risk. Pediatrics. 2013;131:14-21.

7. American Heart Association. Why should I limit sodium 2017 [cited 2018/04/29]. https://www.heart.org/idc/groups/heart-public/@wcm/@hcm/documents/downloadable/ucm_300625.pdf. Accessed April 29, 2018.

8. World Health Organization. WHO issues new guidance on dietary salt and potassium. [cited 2018/04/29]. http://www.who.int/ mediacentre/news/notes/2013/salt_potassium_20130131/en/. Accessed April 29, 2018.

9. Visser FW, Krikken JA, Muntinga JH, et al. Rise in extracellular fluid volume during high sodium depends on BMI in healthy men. Obesity. 2009;17:1684-1688.
10. Curnow KM, Tusie-Luna M-T, Pascoe L, et al. The product of the CYP11B2 gene is required for aldosterone biosynthesis in the human adrenal cortex. Mol Endocrinol. 1991;5:1513-1522.

11. Yu Z, Eckert GJ, Liu H, et al. Adiposity has unique influence on the renin-aldosterone axis and blood pressure in black children. $J$ Pediatr. 2013;163:1317-1322.

12. Kannel WB, Brand N, Skinner JJ, et al. The relation of adiposity to blood pressure and development of hypertension - The Framingham Study. Ann Intern Med. 1967;67:48-59.

13. Sacks FM, Svetkey LP, Vollmer WM, et al. Effects on blood pressure of reduced dietary sodium and the Dietary Approaches to Stop Hypertension (DASH) diet. N Engl J Med. 2001;344:3-10.

14. Patro BK, Jeyashree K, Gupta PK. Kuppuswamy's socioeconomic status scale 2010-the need for periodic revision. Indian J Pediatr. 2012;79:395-396

15. World Health Organization. Protocol for Population Level Sodium Determination in 24-h Urine Samples. Prepared by WHO/PAHO Regional Expert Group for Cardiovascular Disease Prevention Through Population-Wide Dietary Salt Reduction: Sub-Group for Research and Surveillance. Washington, D.C.: World Health Organization; 2010.

16. World Health Organization. Body mass index - BMI. [cited 2018/02/06]. 2018. http://www.euro.who.int/en/health-topics/ disease-prevention/nutrition/a-healthy-lifestyle/body-mass-index-bmi. Accessed February 6, 2018.

17. Mosteller R. Simplified calculation of body-surface area. N Engl J Med. 1987;317:1098.

18. Shafer KJ, Siders WA, Johnson LK, et al. Validity of segmental multiple-frequency bioelectrical impedance analysis to estimate body composition of adults across a range of body mass indexes. Nutrition. 2009;25:25-32.

19. Wolmarans P, Danster N, Dalton A, et al. Condensed food composition tables for South Africa. Cape Town: South African Medical Research Council; 2010.

20. Takken T, Stephens S, Balemans A, et al. Validation of the Actiheart activity monitor for measurement of activity energy expenditure in children and adolescents with chronic disease. Eur J Clin Nutr. 2010;64:1494.

21. Armstrong T, Bull F. Development of the World Health Organization Global Physical Activity Questionnaire (GPAQ). J Public Health. 2016;14:66-70.

22. Levey AS, Stevens $\mathrm{LA}$, Schmid $\mathrm{CH}$, et al. A new equation to estimate glomerular filtration rate. Ann Intern Med. 2009;150:604-612.

23. Center for Disease Control and Prevention. Sodium Reduction Toolkit: A global opportunity to reduce population-level sodium intake, 2013. [cited 2017/03/05]. https://www.cdc.gov/salt/sodium_toolkit.htm. Accessed March 4, 2017.

24. World Health Organization. Guideline. Sodium intake for adults and children. Geneva. World Health Organization; 2012: 2.

25. Adler AC, Nathanson BH, Raghunathan K, et al. Misleading indexed hemodynamic parameters: the clinical importance of discordant BMI and BSA at extremes of weight. Crit Care. 2012;16:471.

26. Tobiasen J, Hiebert JM, Edlich RF. A practical burn severity index. J Burn Care Res. 1982;3:229-232.

27. Linz P, Santoro D, Renz W, et al. Skin sodium measured with $23 \mathrm{Na}$ MRI at 7.0 T. NMR Biomed. 2015;28:54-62.

28. Hofmeister LH, Perisic S, Titze J. Tissue sodium storage: evidence for kidney-like extrarenal countercurrent systems? Pflugers Arch. 2015;467:551-558.

29. Machnik A, Neuhofer W, Jantsch J, et al. Macrophages regulate salt-dependent volume and blood pressure by a vascular endothelial growth factor-C-dependent buffering mechanism. Nat Med. 2009:2(15):545-552.

30. Schafflhuber M, Volpi N, Dahlmann A, et al. Mobilization of osmotically inactive $\mathrm{Na}+$ by growth and by dietary salt restriction in rats. Am J Physiol Renal Physiol. 2007;292:1490-1500. 
31. Gruber J, Frakes M. Does falling smoking lead to rising obesity? J Health Econ. 2006;25:183-197.

32. Chun I, Park J, Han M-A, et al. The association between smoking, alcohol intake, and low-salt diet: results from the 2008 community health survey. J Korean Diet Assoc. 2013;19:223-235.

33. Duarte-Salles T, Mendez MA, Pessoa V, et al. Smoking during pregnancy is associated with higher dietary intake of polycyclic aromatic hydrocarbons and poor diet quality. Public Health Nutr. 2010;13:2034-2043.

34. Klebanoff MA, Levine RJ, Clemens JD, et al. Serum cotinine concentration and self-reported smoking during pregnancy. Am J Epidemiol. 1998;148:259-262.

35. Goodfriend TL, Egan BM, Kelley DE. Aldosterone in obesity. Endocr Res. 1998;1998(24):789-796.

36. Sowers JR, Zemel MB, Zemel P, et al. Salt sensitivity in blacks. Salt intake and natriuretic substances. Hypertension. 1988;12:485-490.

37. Kant AK, Graubard BI, Kumanyika SK. Trends in black-white differentials in dietary intakes of US adults, 1971-2002. Am J Prev Med. 2007;32:264-272.

38. Wagner DR, Heyward VH. Measures of body composition in blacks and whites: a comparative review. Am J Clin Nutr. 2000;71:1392-1402.
39. Tooze JA, Krebs-Smith SM, Troiano RP, et al. The accuracy of the Goldberg method for classifying misreporters of energy intake on a food frequency questionnaire and 24-hour recalls: comparison with doubly labeled water. Eur J Clin Nutr. 2012;66:569.

\section{SUPPORTING INFORMATION}

Additional supporting information may be found online in the Supporting Information section at the end of the article.

How to cite this article: Crouch SH, Ware LJ, GafaneMatemane LF, et al. Dietary sodium intake and its relationship to adiposity in young black and white adults: The African-PREDICT study. J Clin Hypertens. 2018;20:11931202. https://doi.org/10.1111/jch.13329 